\title{
DINÂMICA DO CARBONO EM UMA FLORESTA MANEJADA CO MERCIALMENTE NO AMAZONAS
}

\author{
Rosiele dos Santos Vasconcelos ${ }^{1 *}$, Celso Paulo de Azevedo², Cíntia Rodrigues de Souza², \\ Mabiane Batista França Carreire ${ }^{3}$, Elias Lourenço Vasconcelos Neto ${ }^{4}$ \\ ${ }^{1}$ Universidade Federal do Amazonas, Manaus, Amazonas, Brasil - rosi.engflorestal@ hotmail.com* \\ ${ }^{2}$ Embrapa Amazônia Ocidental, Manaus, Amazonas, Brasil - celso.azevedo@embrapa.br; cintia.souza@embrapa.br \\ ${ }^{3}$ Faculdade Metropolitana de Manaus, Manaus, Amazonas, Brasil - mabiane@ hotmail.com \\ ${ }^{4}$ Universidade Nilton Lins, Manaus, Amazonas, Brasil - netoelvn@gmail.com \\ Recebido para publicação: 13/08/2015 - Aceito para publicação: 28/04/2016
}

\begin{abstract}
Resumo
O objetivo deste estudo foi avaliar os efeitos do sistema silvicultural policíclico na dinâmica de carbono de uma floresta manejada comercialmente no Amazonas. Os dados são provenientes de medições de 41 parcelas permanentes, um hectare cada, localizadas nas Unidades de Produção Anual B, C e D da Fazenda Dois Mil pertencente à Empresa Mil Madeiras Preciosa Ltda, em Itacoatiara, AM. Sendo quatro medições na UPA B (1996, 1998, 2001 e 2014) e três nas UPAs C (1997, 2001 e 2014) e D $(1998,2001$ e 2014), onde todas as árvores com $\mathrm{DAP} \geq 15 \mathrm{~cm}$ foram medidas. Foram avaliadas as variáveis: classe de identificação do fuste, tratamento silvicultural, iluminação e forma da copa, dano e presença de cipó. A biomassa foi estimada utilizando-se uma equação ajustada para floresta tropical úmida. O estoque de carbono estimado foi de 165,7 t.ha ${ }^{-1}$. O incremento periódico anual em carbono é influenciado pelas variáveis categóricas: iluminação da copa e forma da copa. Nas UPAs B e D, 17 e 16 anos após exploração, o carbono é diferente do estoque antes da exploração. A UPA C apresenta o mesmo estoque de carbono que continha antes da exploração, considerado o povoamento total. De modo geral, o manejo aplicado não influenciou de maneira diferente as UPAs, a diferença nos estoque foi somente em função do tempo.

Palavras-chave: Amazônia; manejo florestal; mudanças climáticas.
\end{abstract}

\begin{abstract}
Evaluation of the carbon Dynamics in a commercial managed forest in Amazonas. This study aimed to evaluate the effects of a polycyclic silvicultural system, in the stock and carbon dynamics of a commercial managed forest in Amazonas. Data used in this study are from 41 permanent plots of one hectare each, located in Annual Production Units B, C and Dby the Farm Dois Mil belonging to company Mil Madeiras Preciosa Ltda It is four measurements in UPA B (1996, 1998, 2001 and 2014) and three in UPAs C (1997, 2001 and 2014) and D (1998, 2001 and 2014), every tree with $\mathrm{DBH} \geq 15 \mathrm{~cm}$ was had its diameter measured. Also the following variables were measured: stem identification class, silvicultural treatment, canopy lighting, form of canopy, damage and the presence of lianas. For biomass quantification it was used equation to humid rainforest. Carbon stock above ground estimated is 165.7 t.ha $^{-1}$. The annual periodic increment in carbon is influenced by categorical variables crown lighting and form of canopy. In UPAs B and D, 17 and 16 years after logging the carbon is different from the stock before the logging. The UPA C features the same carbon stock that contained before the logging, considering the total population. In general, the management applied not influenced differently UPAs, the difference in the stock was only a function of time.

Keywords: Amazon; forest management; climatic changes.
\end{abstract}

\section{INTRODUÇÃO}

A floresta amazônica brasileira fornece serviços ambientais à região amazônica, ao Brasil e ao mundo como um todo. Estes serviços podem ser agrupados em manutenção de biodiversidade, ciclo hidrológico e armazenamento de carbono. A partir desse conceito a interação entre floresta e clima tem se destacado dos demais e passou a ser dimensionado e valorado. (FEARNSIDE, 2008).

A importância da floresta amazônica tem se tornado ainda mais evidente quanto ao controle das mudanças climáticas globais, tanto pela capacidade de emitir gases do efeito estufa para a atmosfera, via queimadas ou desmatamentos, como de absorver carbono da atmosfera por meio do crescimento do povoamento (SOUZA, 2012). 
As mudanças climáticas têm causado impactos sobre os sistemas naturais e humanos em todos os continentes e através dos oceanos, principalmente nas últimas décadas, porém as evidências de impactos de mudanças climáticas são mais fortes e mais abrangentes para os sistemas naturais. Resultados dessas mudanças foram divulgados no quinto Relatório de Avaliação do Clima do Painel Intergovernamental de Mudanças Climáticas (INTERGOVERNMENTAL PANEL ON CLIMATE CHANGE (IPCC)), divulgado em 2013, trouxe ainda mais certeza sobre a contribuição das atividades antrópicas nas mudanças climáticas que estão ocorrendo no planeta. Os cientistas concluíram que o homem, com $95 \%$ de certeza, está contribuindo com a maior parte do aquecimento global.

Em 2007, o Painel Intergovernamental sobre Mudança do Clima (IPCC) divulgou em seu relatório sobre o aquecimento global que, na área florestal, existem quatro opções de mitigação dos efeitos das mudanças climáticas: manejo florestal, redução do desmatamento, florestamento e reflorestamento. No curto prazo, os benefícios da mitigação por meio do manejo florestal e do desmatamento evitado são maiores do que os benefícios do reflorestamento e do florestamento.

Segundo Santos (1996), o manejo adequado dos povoamentos florestais é uma alternativa de mitigação importante para que os mesmos sejam transformados em sequestradores de carbono, sendo esta a principal alternativa para a área remanescente da floresta amazônica. As técnicas de manejo florestal devem ser aplicadas de formas adequadas e aliadas ao desenvolvimento dessas técnicas, pesquisas devem ser desenvolvidas para um melhor entendimento do processo de recuperação.

O manejo florestal é fundamental como forma de reconhecer a necessidade de manutenção das florestas tropicais, tornando-o competitivo economicamente, invertendo a relação de valor entre as florestas e a agricultura e pecuária, e assim minimizar o desmatamento na região amazônica (SCHWARTZ et al., 2012).

Diversos estudos avaliam se as florestas tropicais não manejadas são consideradas fonte ou sumidouro de carbono. Por um lado, a floresta pode agir como sumidouro, pois as árvores absorvem carbono durante a fotossíntese e estocam o excesso na forma de biomassa (MALHI et al., 1998; PHILLIPS et al., 1998; NOBRE, 2001; HIGUCHI et al., 2004). Porém, as florestas também podem ser fonte de carbono, devido à diminuição da produtividade florestal e aumento nas taxas de mortalidade decorrentes do aumento da temperatura e da severidade das secas, principalmente causadas pelo fenômeno El Niño (CLARK, 2004), ou devido ao desmatamento, fonte significativa de gases de efeito estufa para a atmosfera (FEARNSIDE, 1997; HOUGHTON et al., 2000).

Diferentes segmentos da sociedade reconhecem a vocação florestal da região amazônica e consideram ser possível conciliar seu desenvolvimento com a conservação de seu imenso patrimônio natural. Para o aproveitamento adequado dos recursos existentes, é necessário antes de tudo avaliar a dinâmica da floresta, procedendo ao seu monitoramento que resulta na obtenção de uma série de informações sobre sua dinâmica. $\mathrm{O}$ entendimento do comportamento da floresta tropical após a exploração é de extrema importância para orientar o seu manejo. Atualmente existe uma demanda grande para este tipo de informação, tendo em vista a necessidade de alternativas para o desenvolvimento sustentável da região em um cenário de mudanças climáticas (AZEVEDO, 2006).

Nesse aspecto, o presente estudo tem como objetivo avaliar os efeitos de curto e médio prazo do sistema silvicultural policíclico, no estoque e dinâmica de carbono de uma floresta de terra firme no estado do Amazonas.

\section{MATERIAL E MÉTODOS}

\section{Localização e Caracterização da Área}

Este estudo faz parte do TmFO (Tropical managed Forests Observatory ), rede pan-tropical, que estuda os efeitos de longo prazo do manejo florestal nos ecossistemas florestais tropicais. Neste trabalho foram inventariadas parcelas permanentes de propriedade da empresa Mil Madeiras Preciosas Ltda., localizada no município de Itacoatiara - Amazonas, a $227 \mathrm{~km}$ a leste de Manaus. A área florestal está localizada entre as latitudes $2^{\circ} 43^{\prime}$ e $3^{\circ}$ 04' S e longitudes $58^{\circ} 31^{\prime}$ e $58^{\circ} 57^{\prime}$ W. A área de estudo corresponde a uma floresta tropical fechada de terras baixas da sub-região de baixos platôs da Bacia Amazônica (RADAMBRASIL, 1978). Na classificação de Köppen o clima da área caracteriza-se por ser chuvoso, do tipo Amw (chuvas do tipo monções). A precipitação pluviométrica é de cerca de $2.200 \mathrm{~mm}$, diminuindo bastante nos meses de agosto e outubro, a temperatura média anual é de $26^{\circ} \mathrm{C}$ e a umidade relativa do ar é de $80 \%$ ao longo do ano (RADAMBRASIL, 1978).

\section{Monitoramento da Floresta}

Os dados são provenientes de 41 parcelas permanentes das Unidades de Produção Anual (UPA) B, C e D (Figura 1). A situação das UPAs, com os ano de exploração e medição, números de parcelas e intensidades de cortes podem ser observados na tabela 1 . 


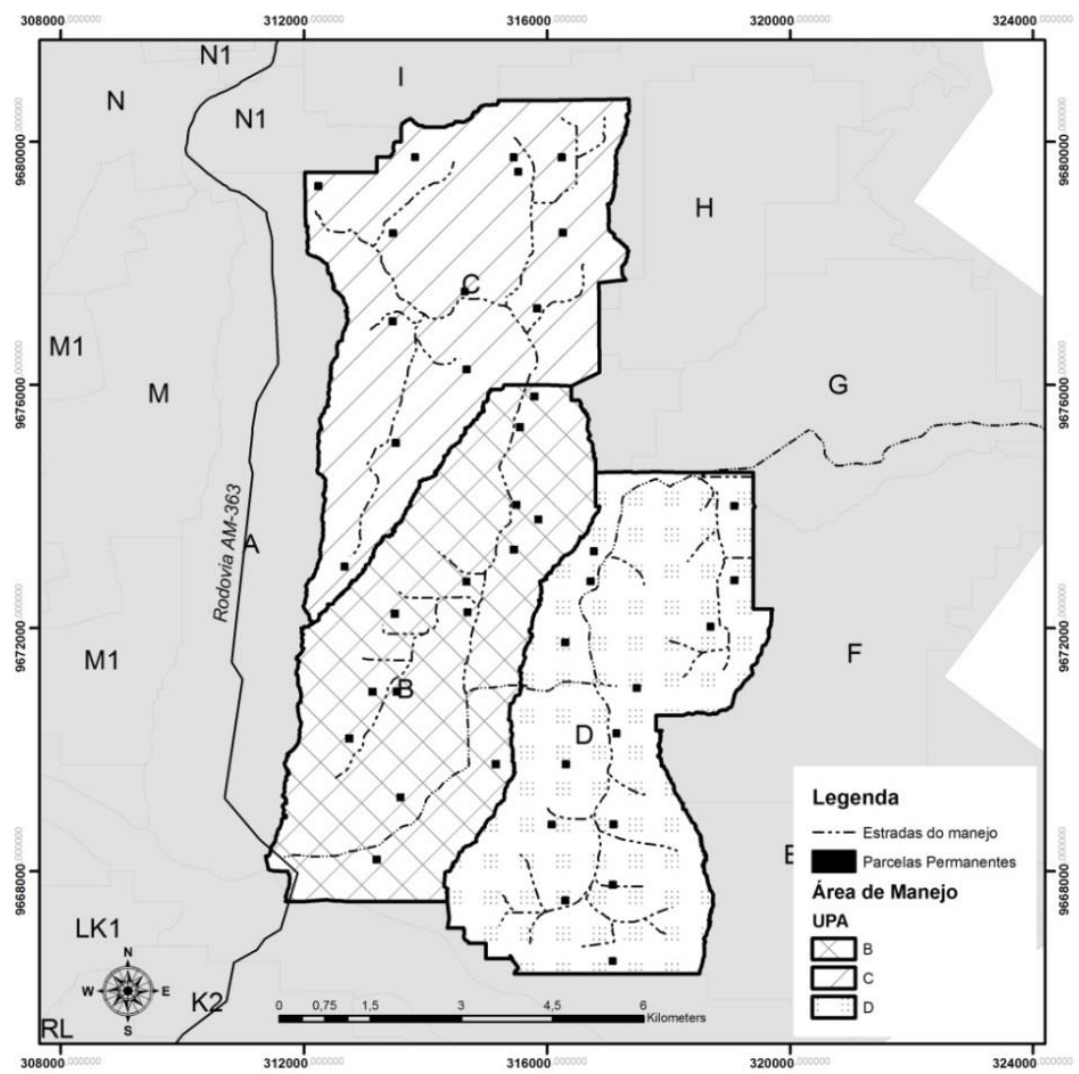

Figura 1. Mapa de distribuição das parcelas permanentes nas Unidades de Produção Anual B, C e D. Fazenda Dois Mil pertencente à Empresa Mil Madeiras Preciosa Ltda. Itacoatiara, AM.

Figure 1. Map of distribution of permanent plots in the Annual Production Units B, C and D. Farm Dois Mil belonging to company Mil Madeiras Preciosa Ltda. Itacoatiara, AM.

Tabela 1. Situação das Unidades de Produção Anual B, C e D. Fazenda Dois Mil pertencente à Empresa Mil Madeiras Preciosa Ltda. Itacoatiara, AM.

Table 1. Status of Annual Production Units B, C and D Farm Dois Mil belonging to company Mil Madeiras Preciosa Ltda. Itacoatiara, AM.

\begin{tabular}{|c|c|c|c|c|c|c|}
\hline Área & Exploração & Medição & Mês & Ano & $\mathbf{N}^{0}$ Parcelas & $\begin{array}{l}\text { Intensidade de } \\
\text { corte }\left(\mathrm{m}^{3} \cdot \mathrm{ha}^{-1}\right)\end{array}$ \\
\hline \multirow{4}{*}{ B } & \multirow{4}{*}{$1996 / 1997$} & 01 & Dez & 1996 & \multirow{4}{*}{14} & \multirow{4}{*}{20,77} \\
\hline & & 02 & Mai & 1998 & & \\
\hline & & 03 & Mar & 2001 & & \\
\hline & & 04 & $\mathrm{Ag}$ & 2014 & & \\
\hline \multirow{3}{*}{$\mathrm{C}$} & \multirow{3}{*}{1997} & 01 & Out & 1997 & \multirow{3}{*}{13} & \multirow{3}{*}{16,66} \\
\hline & & 02 & Ago & 2001 & & \\
\hline & & 03 & Fev & 2014 & & \\
\hline \multirow{3}{*}{$\mathrm{D}$} & \multirow{3}{*}{1998} & 01 & Abr & 1998 & \multirow{3}{*}{14} & \multirow{3}{*}{22,64} \\
\hline & & 02 & Out & 2001 & & \\
\hline & & 03 & Nov & 2014 & & \\
\hline
\end{tabular}

As parcelas foram implantadas com área de um hectare cada $(100 \mathrm{~m}$ x $100 \mathrm{~m})$ conforme a metodologia adotada por Silva e Lopes (1984). Cada parcela foi dividida em 100 subparcelas de $10 \mathrm{~m}$ x $10 \mathrm{~m}$. Todos os indivíduos arbóreos com Diâmetro à Altura do Peito (DAP) igual ou superior a $15 \mathrm{~cm}$ foram identificados e medidos.

As árvores foram identificadas botanicamente em nível de espécie, e os nomes botânicos apresentados foram conferidos com o banco de dados público do Missouri Botanical Garden. Cada indivíduo foi avaliado a partir das variáveis categóricas: Classificação e Identificação do Fuste-CIF (1-Árvore viva em pé com o fuste

FLORESTA, Curitiba, PR, v. 46, n. 2, p. 197 - 205, abr. / jun. 2016

Retslaff, F. A. de S. et al.

ISSN eletrônico 1982-4688

DOI: $10.5380 /$ rf.v46i2.38301 
completo; 2-Árvore viva em pé, sem copa, fuste $\geq 4,0 \mathrm{~m}$ de comprimento; 3-Árvore viva em pé, sem copa, $\leq$ 4,0 m de comprimento; 5-Árvore viva caída); Iluminação da Copa (1-Copa emergente; 2-Copa parcialmente iluminada; 3-Copa completamente coberta por copas de árvores vizinhas; 4-Sem copa); Forma da Copa (1Copa bem distribuída; 2-Copa completa irregular; 3-Copa incompleta; 4-Rebroto; 5-Sem copa); e Presença e Efeitos dos Cipós (1-Nenhum cipó na árvore; 2-Presentes, sem causar danos; 3-Presentes, restringindo o crescimento; 4- Cortados, vivos, sem causar danos; 5-Cortados, vivos, restringindo o crescimento; 6-Cortados e mortos). Essa classificação segue as diretrizes de Silva et al. (2005).

\section{Análise dos Dados}

Dinâmica do carbono (2014):

Para determinação da biomassa seca acima do solo, foi utilizada a equação proposta por Chave et al.

$$
A G B_{e s t}=e^{\left(1,803-0,976 E+0,976 \ln (\rho)+2,673 \ln (D)-0,0299\left(\ln (D)^{2}\right)\right.}
$$

em que: $\mathrm{AGB}_{\text {est }}=$ Biomassa acima do solo; $\mathrm{P}=$ Densidade básica $\left(\mathrm{g} / \mathrm{cm}^{3}\right) ; \mathrm{Ln}=$ Logaritmo na base natural (logaritmo neperiano); $\mathrm{d}=\mathrm{DAP}(\mathrm{cm}) ; \mathrm{E}=(0,178 \mathrm{TS}-0,938 \mathrm{CWD}-6,61 \mathrm{PS}) 10^{-3} ; \mathrm{TS}=$ Temperatura média do período; CWD = Déficit Hídrico Climatológico; PS = Precipitação média do período.

Segundo estudos de Higuchi e Carvalho (1994), 50\% do peso seco de uma árvore correspondem ao carbono estocado. O mesmo valor foi encontrado por Houghton et al. (2000) em estudos realizados na Amazônia Legal. Logo, neste trabalho considerou-se que o conteúdo de carbono correspondea $50 \%$ do teor de biomassa seca.

$$
C=A G B_{\text {est }} \times 0,5
$$

em que: $\mathrm{C}=$ Carbono acima do solo; $\mathrm{AGB}_{\mathrm{est}}=$ Biomassa acima do solo; $0,5=$ Porcentagem correspondente ao estoque de carbono.

Para avaliar a dinâmica de carbono da floresta tropical em estudo, foi efetuado o cálculo do incremento periódico anual em carbono (IPA $\mathrm{C}_{\mathrm{C}}$. Estes valores foram calculados para todas as espécies, com evidência para as mudanças no tempo e comparações entre períodos de tempo pré e pós-exploração.

A fórmula para o cálculo do Incremento Periódico Anual em Carbono (IPA $A_{C}$ é a seguinte:

$$
\begin{gathered}
\text { Crescimento }_{21}=C_{2}-C_{1} \\
\text { Nanos }_{21}=\text { ANO }_{2}-A N O_{1} \\
\text { Nmes }_{21}=\text { MES }_{2}-M E S_{1} \\
\text { Intervaloano }_{21}=\text { Nanos }_{21}+\frac{\text { Nmes }_{21}}{12} \\
\text { IPAC }_{21}=\frac{\text { Crescimento }_{21}}{\text { Intervalo ano }_{21}}
\end{gathered}
$$

em que: $\mathrm{C}_{2}$ e $\mathrm{C}_{1}=$ Teor de carbono no final e no início do período, respectivamente; Crescimento ${ }_{21}=$ Crescimento em teor de carbono do período; $\mathrm{ANO}_{2}$ e $\mathrm{ANO}_{1}=$ Anos no final e no início do período, respectivamente; $\mathrm{Nanos}_{21}=$ Número de anos no período; Mês ${ }_{2}$ e Mês $s_{1}=$ Mês no final e no início do período, respectivamente; Intervalo ano $_{21}=$ Intervalo em anos no período; IPAC $21=$ Incremento Periódico Anual em Carbono.

O ingresso e a mortalidade relativos em carbono foram obtidos pelas seguintes equações:

$$
\begin{aligned}
R \% & =\frac{\text { Carbono. árv. ingressaram }}{\text { Carbono. árv. total da medição anterior }} \times 100 \\
M \% & =\frac{\text { Carbono. árv. morreram }}{\text { Carbono. árv. total da medição anterior }} \times 100
\end{aligned}
$$

em que: $\mathrm{R} \%$ = Taxa de recrutamento em carbono; $\mathrm{M} \%$ = Taxa de mortalidade em carbono

\section{Testes estatísticos}

Foi realizada análise de variância(ANOVA) para o incremento periódico anual em carbono em função das seguintes variáveis: iluminação da copa e forma de copa, considerando os diferentes níveis de classificação como tratamento. Atendendo-se os pressupostos da ANOVA foram realizados os testes de Bartlett e Shapiro- 
Wilk, para verificar a homocedasticidade da variância e a normalidade da distribuição dos erros, respectivamente ao nível de $5 \%$ de probabilidade.

Para observar o efeito da exploração florestal na comunidade remanescente nos estoque de antes e após exploração foi realizado o estudo do Teste $\mathrm{t}$ de Student a $5 \%$ de probabilidade, realizado para amostras independente (considerando o recrutamento e mortalidade) e pareadas, onde se considerou os estoques dos indivíduos que estavam presentes antes da exploração e que permaneceram após exploração.

Análises de variância com medidas repetidas no tempo (VON ENDE, 2001) foram realizadas para avaliar se os estoques em carbono foram influenciados pelas diferentes UPAs exploradas, com o passar do tempo. Para esta análise foram considerados os anos de 2001 e 2014 para os seguintes estoques: Estoque de carbono total (considerando ingresso e mortalidade) e estoque de indivíduos que estavam presentes no ano de 2001 e que permaneceram presentes em 2014 (sem considerar ingresso e mortalidade). A análise foi conforme o seguinte modelo experimental:

$$
X_{j k l}=\mu+T_{j}+B_{k}+T B_{j k}+\varepsilon_{m(j k)}
$$

em que: $\mathrm{T}=$ Tratamento $\mathrm{j}=1, \ldots, \mathrm{p} ; \mathrm{B}=$ Tempo $(\mathrm{T}) \ldots \ldots \ldots \mathrm{k}=1, \ldots, \mathrm{q} ; \mathrm{X}_{\mathrm{jkl}}=$ A observação correspondente ao j-ésimo tratamento e k-ésimo tempo; $\mu=$ Média geral comum a todas as observações; $T_{j}=$ Efeito do j-ésimo tratamento fator fixo; $\mathrm{B}_{\mathrm{k}}=$ Efeito do k-ésimo tempo - fator fixo; $\varepsilon=$ Erro aleatório associado à observação $\mathrm{X}_{\mathrm{jkl}}-$ fator aleatório.

A análise de variância para medidas repetidas foi realizada por meio software estatístico SAS 9.2 licenciado para a EMBRAPA.

\section{RESULTADOS E DISCUSSÕES}

$\mathrm{Na}$ área estudada foram inventariados 12.640 indivíduos, compostos por 184 espécies, pertencentes a 45 famílias botânicas.

A média do estoque de carbono acima do nível do solo estimado para área em estudo é de $165,7 \pm 24,1$ t.ha $^{-1}$. Para a Amazônia, diversos autores encontraram valores de carbono acima do nível do solo próximos aos valores deste estudo, mesmo considerando diferentes métodos de cálculo da biomassa. Keller et al. (2001), trabalhando na Floresta Nacional de Tapajós, estimaram uma biomassa acima do solo, para DAP > 35 $\mathrm{cm}$, de 372 t.ha $^{-1}$, oque corresponde a um estoque de carbono de 186 t.ha ${ }^{-1}$.O trabalho realizado por Lima (2010), avaliando o Sistema de Inventario Florestal Contínuo (IFC) do laboratório de manejo florestal do Instituto Nacional de Pesquisa da Amazônia (INPA), apresenta uma lista de 16 sítios no estado do Amazonas com os valores de carbono acima do solo variando de 99,4 t.ha ${ }^{-1}$ em uma área manejada de Lábrea, a 169,86 t.ha ${ }^{-1} \mathrm{em}^{-}$ uma área em Manaus.

Fearnside (2000) estimou a biomassa total na Amazônia brasileira (considerando-se a biomassa acima e abaixo do solo) em valores entre 433,6 e 464 t.ha ${ }^{-1}$ em média, que corresponde a 216,8 e 232 t.ha $^{-1}$ de carbono, considerando as florestas densas e não densas. Saatchi et al. (2007) utilizando dados de 216 parcelas permanentes instaladas em áreas de terra-firme na região amazônica, estimaram o carbono médio destas florestas em 127,4 t.ha ${ }^{-1}$.

As taxas anuais de recrutamento e mortalidade em carbono foram em média 2,94\% e $0,38 \%$, respectivamente. As maiores taxas de mortalidade em carbono foram observadas logo após a exploração, essas taxas são decorrentes da mortalidade natural, e dos danos às árvores remanescentes em consequência da exploração (Tabela 2). As taxas de mortalidade e recrutamento definem a dinâmica do carbono da vegetação. Normalmente, o diâmetro das árvores mortas, e consequentemente o carbono presente das árvores mortas, é sempre maior do que o das recrutas, por essa razão, a taxa de recrutamento foi menor do que a taxa de mortalidade em carbono. Para cada UPA é possível observar que com o passar do tempo há um aumento da taxa de recrutamento.

A área em estudo apresentou valores de Incremento Periódico Anual em Carbono (IPA ${ }_{C}$ ) que variaram de 1,6 a 2,7 tha ${ }^{-1}$. ano ${ }^{-1}$ (Tabela 2). De acordo com Oliveira (2010) valores semelhantes de IPA IPram $_{C}$ encontrados na reserva de desenvolvimento sustentável de Anamã, onde o incremento periódico anual em carbono variou entre 2,4 a $3,1 \mathrm{t} \mathrm{ha}^{-1}$ ano $^{-1}$, porém quando comparado com outros resultados, o mesmo é considerado alto, já que de acordo com Philips et al. (1998) a média de acumulação de carbono na Amazônia, é igual a $0,97 \mathrm{t} \mathrm{ha}^{-1}$ ano $^{-1}$, estimada com base em 97 parcelas permanentes.

Souza (2012) encontrou valores inferiores em seu estudo realizado em uma área manejada experimentalmente, onde o incremento periódico anual em estoque de carbono variou de 0,3 a 1,56 t.ha $^{-1}$. ano $^{-1}$. $\mathrm{O}$ incremento encontrado nesse estudo pode ser devido ao crescimento individual das árvores compensando as perdas causadas pela mortalidade e exploração, essa acumulação pode explicar em parte, para onde está indo o "carbono perdido" estimado em 1,6 bilhão t/ano, segundo Houghton (1994). 
Tabela 2. Taxas (\%) anuais de mortalidade e recrutamento em carbono acima do solo e Incremento Periódico Anual em Carbono (IPA ) por intervalos de medição e Unidades de Produção Anual (UPA).

Table 2. Annual rates $(\%)$ of recruitment and mortality in carbono above ground and Annual Increment in Carbon $\left(\mathrm{PAI}_{\mathrm{C}}\right.$ ) by measuring ranges and Annual Production Units (APU).

\begin{tabular}{|c|c|c|c|c|}
\hline UPA & Período & Mortalidade (\%) & Recrutamento (\%) & $\mathrm{IPA}_{\mathrm{C}}\left(\right.$ t.ha $\left.^{-1} \mathrm{ano}^{-1}\right)$ \\
\hline \multirow{3}{*}{ B } & 1996-1998 & 7,21 & 0,35 & 2,7 \\
\hline & 1998-2001 & 2,42 & 0,23 & 2,4 \\
\hline & 2001-2014 & 1,54 & 0,72 & 2,5 \\
\hline \multirow{2}{*}{ C } & $1997-2001$ & 2,60 & 0,08 & 1,6 \\
\hline & $2001-2014$ & 1,83 & 0,62 & 2,2 \\
\hline \multirow{2}{*}{ D } & $1998-2001$ & 3,37 & 0,11 & 2,6 \\
\hline & 2001-2014 & 1,64 & 0,57 & 2,5 \\
\hline
\end{tabular}

A partir dos resultados dos testes de Bartlett e Shapiro-Wilk que observou-se que os pressupostos básicos da estatística estão de acordo para a aplicação do teste de médias. Com o teste de Shapiro-Wilk, pode-se inferir que os resíduos de cada tratamento são provenientes de uma distribuição normal $(\rho>0,05)$. O teste de homogeneidade de variâncias de Bartlett indicou que as variâncias dos dados são homogêneas $(\rho>0,05)$.

Nos resultados das análises de variância do IPA $\mathrm{C}_{C}$ em função das variáveis categóricas, houve diferença

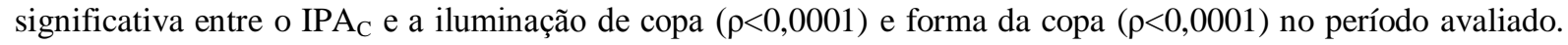
Na comparação das médias pelo teste de Tukey, o maior incremento periódico anual em relação ao carbono ocorreu em árvores com copa completamente exposta à luz. Quanto à forma da copa, o incremento foi maior em árvore que apresentaram copa completa.

Resultados semelhantes foram encontrados por Silva (1989) que avaliou o comportamento do crescimento por classe de iluminação da copa aos oito anos após a exploração e verificou que as árvores que recebiam iluminação total cresceram $30 \%$ mais do que as parcialmente sombreadas e $57 \%$ mais que as árvores totalmente sombreadas.

A fim de avaliar o processo de recuperação da floresta após o manejo, foram feitas comparações da média do estoque de carbono antes da exploração e as médias nos anos em que houve mensurações, essas análises foram realizadas a partir do teste t de Student, tanto para o povoamento total, levando em consideração o recrutamento e mortalidade (Amostras independentes), como também para o povoamento não levando em consideração os dados de recrutamento e mortalidade (Amostras pareadas).

Nas comparações do estoque de carbono contido na floresta antes e após exploração para amostras independentes (Tabela 3), somente os estoque de carbono de 2001 e 2014 na UPA C não apresentaram diferença estatística significativa, ou seja, o conteúdo de carbono é igual estatisticamente às quantidades presentes na floresta antes desta ser explorada. Em relação às UPAs B e D, todas as comparações de antes e após a exploração apresentaram diferença significativa.

Na UPA B um ano após a exploração a diferença no estoque foi significativo a $1 \%$ de probabilidade, 17 anos após a exploração a diferença estatística foi a 5\%, podendo afirmar que apesar de haver diferenças nos estoques, essa UPA apresentou recuperação quanto aos estoques de carbono. Na UPA D, ainda existe diferenças a $1 \%$, porém observa-se que os estoques apresentam tendência à recuperação.

Tabela 3. Comparação das médias pelo teste $t$ de Student entre os estoques de carbono (t.ha ${ }^{-1}$ ) dos anos antes e após exploração, considerando o povoamento total. Em que: Exploração B-1997, e C e D 1998.

Table 3. Comparison of means by $\mathrm{t}$ - test Student's between carbon stocks $\left(\mathrm{t} \mathrm{ha}^{-1}\right)$ the years before and after logging, considering the total population. Wherein: logging B- 1997, C 1998 and D.

\begin{tabular}{lcccc}
\hline UPA & Antes e após exploração & Carbono t.ha $^{-1}$ & T & p \\
\hline \multirow{2}{*}{ B } & $1996 \times 1998$ & $179,1 \times 146,5$ & $3,60^{* *}$ & 0,0013 \\
& $1996 \times 2001$ & $179,1 \times 144,3$ & $3,75^{* *}$ & 0,0009 \\
\hline \multirow{2}{*}{ C } & $1996 \times 2014$ & $179,1 \times 161,1$ & $2,11^{*}$ & 0,0461 \\
\hline \multirow{2}{*}{ D } & $1997 \times 2001$ & $163,5 \times 146,1$ & $1,46^{\text {ns }}$ & 0,1558 \\
& $1997 \times 2014$ & $163,5 \times 151,9$ & $0,95^{\text {ns }}$ & 0,3517 \\
\hline
\end{tabular}

ns - não significativo, *significativo a $5 \%$ de probabilidade, **significativo a $1 \%$ de probabilidade

A comparação de estoque sem levar em consideração o recrutamento e mortalidade (Amostras pareadas) teve como objetivo eliminar a forte influência que principalmente a mortalidade tem sobre a 
produtividade da floresta, pois quando uma árvore de grande porte morre, a substituição da biomassa perdida é lenta. Pela observação da tabela 4, pode-se notar que quando não se considera a mortalidade e o recrutamento, os valores médios por hectare do estoque de carbono é menor do que os valores observados nos cálculos do povoamento total. Houve diferença altamente significativa $(\rho<0,0001)$ das médias de carbono antes e após a exploração para todas as UPAs e intervalos. Indicando assim que o estoque de carbono vem apresentando uma tendência de crescimento com o passar do tempo.

A fixação de carbono na floresta é reportada como, no mínimo, parcialmente resultado de intensivo manejo florestal. Seidl et al. (2007) avaliaram a correlação entre o sequestro de carbono e a produção de madeira de forma sustentável em uma floresta de múltiplo uso na Áustria e concluíram que o sequestro de carbono da atmosfera é maior nas florestas manejadas sustentavelmente do que nas florestas não manejadas. Pode se dizer, dessa forma, que ao desconsiderar os efeitos da mortalidade e do recrutamento, a floresta não esteve em equilíbrio com a atmosfera, agindo assim como sumidouro de carbono.

Devido ao pequeno número nos graus de liberdade, já que foram considerados somente dois períodos de medição, não foram realizadas as correções de Huynh - Feldt (H-F) e Greenhouse - Geisser (G-G) para possíveis violações da pressuposição de esfericidade da Anova de medidas repetidas, pois de acordo com esses autores as correções nos graus de liberdade são realizadas para que esses testes possam ser usados de forma aproximada, mesmo que a condição de esfericidade não seja satisfeita.

Quando considerado o povoamento total, para o fator tempo, posto que o nível de significância associado é 0,0001 (menor que 1\%), pode-se concluir que o estoque de carbono total não é o mesmo nos dois momentos temporais definidos pelo fator tempo. Quanto à interação tempo x UPA, observa-se que essa interação não foi significativa, ou seja, a influência do manejo florestal aplicado foi igual nas diferentes Unidades de Produção Anual (Tabela 5).

Tabela 4. Comparação das médias pelo teste t de Student entre os estoques de carbono (t.ha ${ }^{-1}$ ) dos anos antes e após exploração (amostras pareadas). Em que: Exploração B-1997, e C e D 1998.

Table 4. Comparison of means by $t$ - test Student's between carbon stocks $\left(\mathrm{t}^{\text {ha }}{ }^{-1}\right)$ the years before and after logging (paired samples. Wherein: logging B- 1997, C 1998 and D.

\begin{tabular}{|c|c|c|c|c|}
\hline UPA & Antes e após exploração & Carbono t.ha $^{-1}$ & $\mathbf{T}$ & $\rho$ \\
\hline \multirow{3}{*}{ B } & $1996 \times 1998$ & $104,7 \times 107,5$ & 12,61 & $<0,0001$ \\
\hline & 1996 x 2001 & $104,7 \times 113,1$ & 22,85 & $<0,0001$ \\
\hline & $1996 \times 2014$ & $104,7 \times 144,8$ & 24,52 & $<0,0001$ \\
\hline \multirow{2}{*}{$\mathrm{C}$} & 1997 x 2001 & $106,2 \times 111,2$ & 14,51 & $<0,0001$ \\
\hline & $1997 \times 2014$ & $106,2 \times 139,5$ & 28,06 & $<0,0001$ \\
\hline \multirow{2}{*}{$\mathrm{D}$} & $1998 \times 2001$ & $126,4 \times 133,7$ & 20,13 & $<0,0001$ \\
\hline & $1998 \times 2014$ & $126,4 \times 166,1$ & 32,41 & $<0,0001$ \\
\hline
\end{tabular}

Tabela 5. ANOVA para medidas repetidas para o carbono total, considerando o ingresso e a mortalidade.

Table 5. Repeated-measures ANOVA for total carbon, considering the entry and mortality.

\begin{tabular}{|c|c|c|c|c|c|}
\hline PARCELA & & & & & \\
\hline Fonte variação & GL & SQ & QM & Fcal & Pr $>F$ \\
\hline UPA & 2 & 11437,4169 & 5718,7084 & $6,27^{* *}$ & 0,0044 \\
\hline Erro & 38 & 34644,8187 & 911,7057 & & \\
\hline \multicolumn{6}{|l|}{ SUBPARCELA } \\
\hline Tempo & 1 & 2308,2711 & 2308,2711 & $18,72^{* *}$ & 0,0001 \\
\hline Tempo * UPA & 2 & 427,9157 & 213,9579 & $1,74^{\mathrm{ns}}$ & 0,1901 \\
\hline Erro (tempo) & 38 & 4685,6937 & 123,3077 & & \\
\hline
\end{tabular}

ns - não significativo, *significativo a $5 \%$ de probabilidade, **significativo a $1 \%$ de probabilidade

Resultados semelhantes foram encontrados por Souza (2012) que ao estudar uma floresta manejada experimentalmente, submetida a quatro tratamentos e quatro períodos de medição (2005, 2007, 2009 e 2010) observou que houve uma tendência de aumento do carbono total (considerando recrutamento e mortalidade) com o passar do tempo e quanto a interação tempo x tratamento, o carbono total não sofreu influência significativa do tratamento com o passar do tempo.

$\mathrm{Na}$ tabela 6 pode-se observar os resultados da análise de variância para medidas repetidas para o carbono total considerando os indivíduos que estavam presentes em 2001 e que permaneceram até 2014. Nota-se que o estoque total de carbono para as UPAs foi influenciado pelo passar do tempo, porém a interação tempo e UPA não foi significativa.

FLORESTA, Curitiba, PR, v. 46, n. 2, p. 197 - 205, abr. / jun. 2016.

Retslaff, F. A. de S. et al.

ISSN eletrônico 1982-4688

DOI: $10.5380 /$ rf.v46i2.38301 
Souza (2012)constatou que o carbono variou significativamente com o passar do tempo e que as interações entre tempo e tratamento de redução de área basal foram estatisticamente significativas, ou seja, a aplicação dos diferentes tratamentos de exploração de área basal influenciou no estoque de carbono com o passar do tempo, quando desconsiderou o recrutamento e mortalidade.

Tabela 6. ANOVA para medidas repetidas no tempo para o número total de carbono dos indivíduos vivos em 2001 e que permaneceram vivos em 2014.

Table 6. Repeated measures ANOVA for total carbon of living individuals in 2001 and remained alive in 2014.

\begin{tabular}{|c|c|c|c|c|c|}
\hline \multicolumn{6}{|l|}{ PARCELA } \\
\hline Fonte variação & GL & SQ & QM & Fcal & Pr $>F$ \\
\hline UPA & 2 & 9448,5198 & 4724,2599 & $4,37^{*}$ & 0,0196 \\
\hline Erro & 38 & 41080,9665 & 1081,0780 & & \\
\hline \multicolumn{6}{|l|}{ SUBPARCELA } \\
\hline Tempo & 1 & 20403,7936 & 20403,7936 & $1587,14^{* *}$ & $<0,0001$ \\
\hline Tempo * UPA & 2 & 74,6418 & 37,3210 & $2,90^{\mathrm{ns}}$ & 0,0671 \\
\hline Erro (tempo) & 38 & 488,5166 & 12,8557 & & \\
\hline
\end{tabular}

ns - não significativo, *significativo a $5 \%$ de probabilidade, **significativo a $1 \%$ de probabilidade

\section{CONCLUSÕES}

- Considerando o estoque de carbono das árvores vivas em todas as medições o mesmo apresentou diferença estatística 17 anos após a exploração, caracterizando que a floresta submetida ao manejo florestal funciona com sumidouro, ou seja, sequestrando carbono da atmosfera.

- De maneira geral, o estoque de carbono variou somente em função do tempo (ano), não apresentando diferença em função do manejo aplicado.

\section{REFERENCIAS}

AZEVEDO, C. P. Dinâmica de florestas submetidas a manejo na Amazônia Oriental: experimentação e simulação. 254 f. Tese (Doutorado em Ciências Florestais)-Universidade Federal do Paraná, Curitiba, Paraná.

CHAVE, J.; MÉCHAIN, M. R.; BÚRQUEZ, A.; CHIDUMAYO, E.; COLGAN, M. S.; DELITTI, W. B. C.; DUQUE, A.; EID, T.; FEARNSIDE, P. M.; GOODMAN, R. C.; HENRY, M.; YRÍZAR A. M.; MUGASHA, W.; LANDAU, H. C. M.; MENCUCCINI, M.; NELSON, B. W.; NGOMANDA, A.; NOGUEIRA, E. M.; MALAVASSI, E. O.; PÉLISSIER, R.; PLOTON, P.; RYAN, C. M.; SALDARRIAGA, J. G.; VIEILLEDENT, G. Improved allometric models to estimate the above ground biomass of tropical trees. Global Change Biology, p. 3177 - 3190, 2014.

CLARK, D. A. Sources or sinks? The responses of tropical forests to current and future climate and atmospheric composition. Philosophical Transactions of the Royal Society of London B, 359: 477 - 491, 2004.

FEARNSIDE, P. M. Global warming and tropical land-use change: greenhouse gas emissions from biomass burning, decomposition and soils in forest conversion, shifting cultivation and secondary vegetation. Climatic Change, 46(1-2): 115 - 158. 2000.

FEARNSIDE, P. M. Greenhouse gases from deforestation in Brazilian Amazonia: net committed emissions. Climatic Change, 35(3): 321 - 360, 1997.

FEARNSIDE, P. M. Quantificação do serviço ambiental do carbono nas florestas amazônicas brasileiras. Oecologia Brasiliensis, 12 (4): 743 - 756, 2008.

HIGUCHI, N.; CARVALHO JR., J. A Fitomassa e conteúdo de carbono de espécies arbóreas da Amazônia. In: Seminário emissão x sequestro de $\mathrm{CO}_{2}$ - uma oportunidade de negócios para o Brasil, Rio de Janeiro: CVRD. p. $125-153,1994$.

HIGUCHI, N.; CHAMBERS, J.; SANTOS, J.; RIBEIRO, R. J.; PINTO, A. C. M.; SILVA, R. P. DA; ROCHA, R. DE M.; TRIBUZY, E. S. Dinâmica e balanço do carbono da vegetação primária da Amazônia Central. Revista Floresta, 34 (3): 295 - 304, 2004.

HIGUCHI, N.; PEREIRA, H. S.; SANTOS, J.; LIMA, A. J. N.; HIGUCHI, F. G.; HIGUCHI, M. I. G.; AYRES, I. G. S. Governos locais amazônicos e as questões climáticas globais. Edição dos autores, 104 pp, 2009. 
HOUGHTON, R. A.; SKOLE, D. L.; NOBRE, C. A.; HACKLER, J. L.; LAWRENCE, K. T.; CHOMENTOWSKI, W. H. Annual fluxes of carbon from deforestation and regrowth in the Brazilian Amazon. Nature, 403: 301 - 304, 2000.

INTERGOVERNMENTAL PANEL ON CLIMATE CHANGE (IPCC). 2007. Intergovernmental Panel on Climate Change. Contribución del Grupo de Trabajo III al Cuarto Informe de Evaluación del Grupo Intergubernamental de Expertos sobre Cambio Climático. Resumo Técnico, Disponível em <http://www,ipcc,ch>, Acesso em 02 Jan, 2014.

(IPCC). 2013. Intergovernmental Panel on Climate Change. Working Group I Report "The Physical Science Basis", Resumo técnico,Disponível em<http://www,ipcc,ch〉, Acesso em 15 Jan 2014.

LAMPRECHT, H. Silvicultura nos Trópicos. Eschborn: GTZ, 1990.

LIMA, A. J. N. Avaliação de um sistema de Inventário Florestal Contínuo em áreas manejadas e não manejadas do Estado do Amazonas (AM). Tese (Doutorado em Ciências de Florestas Tropicais). Instituto Nacional de Pesquisas da Amazônia, INPA/UFAM, Manaus, Amazonas, 2010.

MALHI, Y.; NOBRE, A. D., GRACE, J.; KRUIJT B.; PEREIRA M. G. P.; CULF A.; SCOTT S. Carbon dioxide transfer over a Central Amazonian rain forest. Journal of Geophysical Research-Atmospheres, 103 : 31593 - 31612, 1998.

NOBRE, C. A. Amazônia: fonte ou sumidouro de carbono?In: MMA. Causas e dinâmica do desmatamento na Amazônia, Brasília, DF. p. 197 - 224, 2001.

PHILLIPS, O. L.; MALHI, Y.; HIGUCHI, N.; LAURANCE, W. F.; NÚÑEZ, P. V.; VÁSQUEZ, R. M.; LAURANCE, S. G.; FERREIRA, L. V.; STERN, M.; BROWN, S.; GRACE, J. Changes in the carbon balance of tropical forests: evidence from long-term plots. Science, 282: 439 - 442, 1998.

RADAMBRASIL. Propagação de Interação Nacional, Levantamentos de Recursos Naturais. IV- Vegetação, Manaus: DNPM, Ministério das Minas e Energia, v. 18. p. 747, 1978.

SAATCHI, S. S.; HOUGTOHON, R. A.; SANTOS, R. C.; SOARES, J. Distribution of above ground live biomass in the Amazon basin. Global Change Biology, 12: 816 - 837, 2007.

SANTOS, J. Análise de modelos de regressão para estimar a fitomassa da floresta tropical úmida de terrafirme da Amazônia brasileira. 121. Tese (Doutorado em Ciência Florestal)-Universidade Federal de Viçosa, Viçosa, Minas Gerais, 1996.

SCHWARTZ, G.; PEÑA-CLAROS, LOPES, M.; J. C. A.; MOHREN, G. M. J.; KANASHIRO, M. Mid-term effects of reduced-impact logging on the regeneration of seven tree commercial species in the Eastern Amazon. Forest Ecology and Management, 274: 116 - 125, 2012.

SEIDL, R.; RAMMER, W.; JÄGE R. D.; CURRIE, W. S.; LEXER, M. J. Assessing tradeoffs between carbon sequestration and timber production within a framework of multipurpose forestry in Austria. Forest Ecology and Management, 248(1-2): 64 - 79, 2007.

SILVA, J. N. M.; LOPES, J. C. A. Inventário florestal contínuo em florestas tropicais: a metodologia utilizada pela EMBRAPA/CPATU na Amazônia Brasileira. Belém, PA: EMBRAPA-PA, 33 p, 1984.

SIlVA, J. N. M.; LOPES, J. C. A.; OlIVEIRA, L. C.; SILVA, S. M. A.; CARVALHO, J. O. P.; COSTA, D. H. M.; MELO, M. S.; TAVARES, M. J. M. Diretrizes simplificadas para instalação e medição de parcelas permanentes em florestas naturais da Amazônia Brasileira. Belém, PA: Embrapa Amazônia Oriental, 68 p, 2005.

SILVA, J. N. M. The behaviour of the tropical rain forest of the Brazilian Amazon after logging. 302 p. Tese (Doutorado em Ciências Florestais) - University of Oxford, Oxford, 1989.

SOUZA, C. R. Dinâmica de carbono em floresta explorada e em floresta nativa não explorada na Amazônia-Manaus. Tese (Doutorado em Ciências de Florestas tropicais)-Instituto Nacional de Pesquisas da Amazônia (INPA/UFAM), Manaus, Amazonas, 2012.

VON ENDE, C. N. Repetead-Measures Analysis: Growth and Other Time Dependent Measures. In: Scheiner, S. M.; Gurevitch, J. (Eds), Design and Analysis of Ecological Experiments, Oxford University Press, p, 134 $157,2001$. 